\title{
Anabases
}

ANABASES Traditions et réceptions de l'Antiquité

11 | 2010

Varia

\section{Dans le sillage du Rudens : de Plaute à Jules Verne}

Jean Soubiran

\section{OpenEdition}

Journals

Édition électronique

URL : http://journals.openedition.org/anabases/895

DOI : 10.4000/anabases.895

ISSN : 2256-9421

\section{Éditeur}

E.R.A.S.M.E.

\section{Édition imprimée}

Date de publication : 1 mars 2010

Pagination : 227-232

ISSN : 1774-4296

\section{Référence électronique}

Jean Soubiran, «Dans le sillage du Rudens : de Plaute à Jules Verne », Anabases [En ligne], 11 | 2010, mis en ligne le 01 mars 2013, consulté le 21 octobre 2019. URL : http://journals.openedition.org/ anabases/895 ; DOI : 10.4000/anabases.895

Ce document a été généré automatiquement le 21 octobre 2019

(c) Anabases 


\title{
Dans le sillage du Rudens : de Plaute à Jules Verne
}

\author{
Jean Soubiran
}

1 Publié par Hetzel en 1883 dans la série des «Voyages extraordinaires », Kéraban-le-Têtu n'est pas le plus connu des romans verniens, qui ont jadis passionné ma jeunesse. Je n'en connaissais que le titre sans rien savoir du sujet, et ne l'ai découvert que tout récemment, sous la forme d'un livre de poche déniché dans un vide-grenier ${ }^{1}$. Surprise ! Sa lecture m'a rappelé un cours d'agrégation que j'ai fait à Toulouse en 1987-88 sur le Rudens, ma préférée peut-être parmi les comédies de Plaute.

2 Le sujet principal du roman français n'a rien à voir, apparemment, avec la comédie latine, sinon une tonalité d'ensemble à la fois romanesque et comique, pimentée çà et là de péripéties dramatiques - celle du naufrage, en particulier: nous allons y venir. Kéraban, riche négociant ottoman à Constantinople, se partage entre ses bureaux de Péra, sur la rive européenne du Bosphore, et sa luxueuse villa de Scutari, sur la rive asiatique. Mais voici qu'un beau jour le sultan impose un péage à acquitter pour tout franchissement du Bosphore. Refusant catégoriquement de s'y soumettre, Kéraban bien surnommé « le Têtu » - décide de contourner l'obstacle : il joindra ses bureaux et sa demeure... en faisant le tour de la Mer Noire, de la Thrace à l'Anatolie : soit 2800 kilomètres en chaise de poste, car il est allergique à tout modernisme, chemin de fer et bateau à vapeur (p. 43-58). C'est ce contournement de l'antique Pont-Euxin, jalonné de maintes aventures, qui constitue le sujet - fort original et ingénieux, voire cocasse - de notre roman ${ }^{2}$. Mais à cette intrigue principale s'entrelace un thème secondaire, le seul qui nous intéressera ici.

\section{Protagonistes}

3 Kéraban a un neveu, fils adoptif et futur héritier, qui l'accompagnera dans son voyage. Ahmet est « un charmant garçon » de 22 ans (p. 106), bien sous tous rapports, « un peu artiste, un peu poète » (p. 37) ; il est fiancé à la fille d'un ami de Kéraban, le banquier Sélim, d'Odessa ${ }^{3}$ : le mariage est prévu dès la fin du voyage. Très éprise de son futur - 
qui le lui rend bien -, Amasia est une toute jeune (16 ans) fille fort séduisante (éléments de portrait p. 100, 138-147) : « Élégance naturelle, flexibilité de sa taille, grâce de sa démarche ", douce, sentimentale, rêveuse, inquiète pour son fiancé. Elle a une suivante, Nedjeb (éléments de portrait p. 100 sq., 108, 140), "d'origine zingare ", "vive, enjouée, de belle humeur franche et communicative» qui contraste heureusement avec le sérieux de sa maitresse.

Si Kéraban lui-même n'a pas de modèle précis dans le Rudens - tout au plus quelques traits de Démonès, assez souvent bourru (infra n. 11), mais les deux personnages ne se correspondent pas dans l'intrigue -, on reconnaîtra aisément, dans le trio Ahmet/ Amasia + Nedjeb, le trio plautinien Pleusidippe/Palaestra + Ampelisca. Pleusidippe est un jeune Athénien d'excellente famille ( $R d .1197$ sq. de genere summo adulescenti... / ingenuo, Atheniensi) et apparemment assez riche pour voyager d'Athènes à Cyrène ( $R d$. 42) et pour racheter au leno Labrax (Rd. 45) la jeune Palaestra dont il est tombé amoureux au premier regard ( $R d .43 \mathrm{sq}$.). L'Ahmet de Jules Verne lui est absolument superposable : le jeune premier, le futur idéal.

Quant à Palaestra et Ampelisca, ce sont toutes deux de jolies filles séduisantes ( $R d .565$ facie scitula, 894 ambas forma scitula atque aetatula; Palaestra seule : 51 formam uirginis; Ampelisca seule : 415 specie lepida ; 421-424; 463 delicata). Mais elles ne sont visiblement pas égales par la naissance et l'éducation. Alors que Palaestra s'enorgueillit d'être née libre ( $R$ d. 217 libera ego prognata fui), Ampelisca (diminutif familier en -isca= «Vignette »!) est qualifiée d'ancillula (Rd. 74). Elle n'a pas la distinction, la réserve de sa compagne, dont la constante gravité évoque une figure tragique digne d'Euripide. Délurée, quelque peu effrontée, elle se prête à un dialogue vif, voire leste, avec l'esclave Scéparnion ( $R d .415-457)$. C'est elle qui assume la corvée d'eau ( $R d .431-435)$, mais, quand il s'agit de s'expliquer avec la prêtresse, c'est Palaestra seule qui parle. Enfin, une fois libérée, elle épousera le tout juste affranchi Trachalion ( $R d .1220)$. Plus vive, active et hardie, plus expansive que son amie ( $R d .223$ sq., 244 sqq.), Ampelisca est le type même de la soubrette classique (Molière, Marivaux) ${ }^{5}$; et sa ressemblance est patente avec la Nedjeb de Jules Verne. Chez Plaute et le romancier français, les deux «paires » féminines se présentent de manière très semblable.

\section{Rapt}

Une autre particularité, plus rare et par là plus significative encore, relie deux à deux ces quatre jeunes filles. Toutes les quatre ont été victimes d'un rapt qui a jadis bouleversé ou vient de bouleverser leur existence. Sur les antécédents d'Ampelisca nous ne savons rien, sinon qu'elle est au pouvoir du leno Labrax qui s'apprête à faire d'elle une meretrix. Le même sort attend Palaestra ${ }^{6}$, dont le prologue nous informe (Rd. 39-66) que, fille de naissance libre de l'Athénien Démonès, lui-même parfaitement honorable ( $R d .36 \mathrm{sq}$.$) , elle fut enlevée à l'âge de trois ans ( R d .744$ trima) par un pirate (Rd. 40 praedo), puis achetée par le leno Labrax et emmenée à Cyrène pour parfaire son éducation de courtisane raffinée ( $R d .43$ ludo fidicinio). Pleusidippe la vit, s'éprit d'elle et voulut la racheter (30 mines, soit un demi-talent), mais fut berné par le leno avec le concours de son parasite, le vieux scélérat Charmidès: la jeune fille devait être emmenée avec d'autres (dont Ampelisca, $R d$. 52) en Sicile pour s'y prostituer. Pleusidippe l'apprend ( $R d .64$ sq.), se précipite au port... trop tard! Rd. 65 sq. Ad portum adulescens uenit, /illorum nauis longe in altum abscesserat... 
7 Dans le roman de Jules Verne, la fille de Sélim, Amasia, a été, pour son malheur, remarquée à Odessa par le seigneur Saffar, de Trébizonde (p.26), personnage sans scrupules bien résolu dès lors à enlever la jeune fille par n'importe quel moyen. Il confie cette mission à deux fieffés coquins (cf. chez Plaute Labrax et Charmidès), son intendant Scarpante et le capitaine d'un navire maltais, Yarhud (p. 18-28). Ce dernier accoste à Odessa et réussit, par ruse et violence, avec le concours de ses matelots, à se saisir d'Amasia et de Nedjeb et à les embarquer de force. Sélim, survenu (comme Pleusidippe) un instant trop tard, ne peut qu'assister impuissant au départ du bateau toutes voiles dehors (p. 147 sq.).

\section{Tempête et naufrage}

8 Mais avant le terme prévu (Agrigente ici, Trébizonde là) des deux voyages, le mauvais temps va se déchaîner au même moment de l'année. Chez Plaute, l'étoile Arcturus en revendique la responsabilité : $R d .69$ sqq. Increpui hibernum et fluctus moui maritimos. / Nam Arcturus signum sum omnium acerrimum: /uehemens sum exoriens, cum occido uehementior. Le v. 71 suggère (cum occido uehementior) que la tempête accompagne le coucher vespéral de l'étoile (derniers jours d'octobre, début novembre) ${ }^{7}$; si l'on préfère le lever matinal (exoriens), il survient courant septembre et coïncide quasiment avec l'équinoxe. Les deux périodes ${ }^{8}$ sont, selon la météorologie antique, génératrices de tempêtes, orages et averses de grêle. Concordance sans équivoque chez Jules Verne: « Nous sommes à l'équinoxe d'automne » (p. 273).

9 Chez Plaute, la tempête surprend le bateau du leno très peu après le départ de Cyrène : Arcturus est intervenu presque aussitôt après l'appareillage (Rd.68) : il n'y a plus aucun rivage entre Cyrène et la Sicile, et il faut que le naufrage se produise tout près de chez Démonès, qui demeure sur la côte cyrénaïque. Chez Jules Verne, au contraire, la traversée d'Odessa à Trébizonde s'achève : le navire de Yarhud a déjà atteint les bords SE de la Mer Noire, entre Batoum et Trébizonde, près du petit port d'Atina (cf. carte p. 85) $)^{9}$.

10 Plaute ne brosse pas un tableau grandiose de la tempête : notations brèves et éparses (infra), vocabulaire assez banal. Ce topos poétique ressortit plutôt à l'épopée (Homère, Virgile, Ovide, Lucain, Val. Flaccus...) et à la tragédie (Euripide, Pacuvius, Sénèque) ${ }^{10}$; Plaute l'abandonne implicitement à cette dernière en renvoyant à l'Alcmène d'Euripide (Rd. 86). En revanche, Jules Verne annonce de loin le cataclysme et multiplie les notations terrifiantes dans un crescendo soigneusement ménagé (p. 266, 272, 281, 291-301), dont le style paroxystique rappelle Sénèque ( $\mathrm{Ag}$.) et Lucain (Ph. V).

11 Pendant que la tartane de Yarhud, qui emporte les jeunes filles, lutte contre les éléments déchaînés, il se trouve que, par une coïncidence extraordinaire, Kéraban et Ahmet sont précisément parvenus, dans leur voyage terrestre, au même point de cette côte turque, et ont dû, devant la tourmente, chercher refuge dans la fragile maisonnette d'un gardien de phare (p. 282 sq.), d'où ils assistent, anxieux et impuissants, au déchaînement croissant de l'ouragan, qu'accompagnent une trombe et des vagues énormes. Et ici, un détail significatif coïncide dans nos deux textes: la maison de Démonès, chez Plaute, vient d'avoir sa toiture emportée - l'incident est rappelé à plusieurs reprises $(R d .78,85,101$ sq., 123, 154). Or Jules Verne attribue les mêmes dégâts à la maisonnette du phare : toit " effondré ", « arraché ", " défoncé " par la chute du pylône qui le surmontait (p. 302 sq.). 


\section{Sauvetage} inévitable. Mais par une chance invraisemblable, les deux jeunes filles - et aussi (infra) leurs ravisseurs - échappent seules à la mort. Pas un mot, en revanche, dans nos deux textes, sur le sort des matelots, qui semblent s'être volatilisés! Quant au détail de ces circonstances dramatiques, il diffère quelque peu d'une oeuvre à l'autre.

(e) Rudens, selon les annonces du prologue (Rd.72-77), les observations de Démonès et Scéparnion ( $R d .148-180$ ) et les souvenirs d'Ampelisca ( $R d .365-372)$, le navire a fait naufrage dans la nuit en se fracassant contre les rochers. Les deux filles se sont jetées dans une chaloupe et, après avoir été longtemps ballottées par les vagues, ont réussi, exténuées et trempées, à prendre pied sur le rivage, sous les yeux de Démonès et Scéparnion qui contemplent le spectacle sans un geste d'assistance, dont Démonès, étrangement dur (s'il savait qui est en perdition à quelques pas de lui !), dissuade son esclave $^{11}$. D'abord séparées, Palaestra et Ampelisca se retrouvent bien vite, se lamentent sur leur sort (deux arias symétriques) et se réfugient dans le temple de Vénus où les réconforte la vieille prêtresse Ptolémocratia (Rd. 258-289), tandis que Labrax et Charmidès, sauvés eux aussi, se morfondent sur un rocher battu des vagues ( $R d .72$ sq.).

Chez Jules Verne, Kéraban, Ahmet et le gardien du phare, ainsi que quelques pêcheurs du voisinage (p. 303) «accourus, peut-être pour se disputer les débris de cette tartane que le ressac allait bientôt rejeter sur les rochers » (le romancier pense-t-il au fameux choeur des pêcheurs du Rudens, 290-305, et à l'avidité de Gripus cramponné à la valise qu'il vient de repêcher?), ont entendu depuis leur fragile abri les coups de canon du navire en détresse (p. 296, 301), puis les cris désespérés d'Amasia qui clame le nom d'Ahmet (p. 306), juste avant que la tartane ne se brise sur les rochers (p. 307). En pleine nuit, "à la lueur des éclairs continus qui illuminaient l'espace», le jeune homme s'élance dans les vagues et réussit à tirer de l'eau les deux naufragées pantelantes. Retrouvailles combien inattendues! car Ahmet ignorait le rapt d'Amasia qu'il avait laissée à Odessa (p. 310): effusions, explications... Mais le ravisseur Yarhud, "seul survivant de son équipage ", en est réchappé aussi ; "sans avoir été vu... il disparaît dans une direction opposée... Il savait maintenant que, par une fatalité inconcevable, Ahmet s'était trouvé sur le lieu du naufrage » (p. 310).

\section{Épilogue}

Comme Ahmet et Amasia, Pleusidippe et Palaestra se retrouvent, mais Plaute (ou son modèle grec Diphile) n'a pas cru bon, ou possible, de mettre en scène le dialogue pathétique des deux jeunes gens miraculeusement réunis : les deux rôles étaient peutêtre joués par le même acteur, et du reste, dans la Néa comme chez Plaute, les uirgines ne jouent qu'un faible rôle dans l'action dont elles sont seulement l'enjeu passif. Plus d'un lecteur moderne le regrettera.

Le parallélisme des deux aventures s'arrête ici. Les deux derniers actes ${ }^{12}$ du Rudens amènent, grâce à la trouvaille par Gripus d'une valise appartenant au leno et contenant de menus objets révélateurs ( $\gamma v \omega \rho i ́ \sigma \mu \alpha \tau \alpha)$, l'identification de Palaestra comme la fille de Démonès. Pleusidippe, donc, l'épousera, tandis qu'Ampelisca, rachetée au leno, 
s'unira à Trachalion, affranchi par Pleusidippe. Chez Jules Verne, le seigneur Saffar et ses deux acolytes n'ont pas renoncé : un autre rapt sera à deux doigts de réussir, mais échouera finalement et tous trois y perdront la vie (p. 422-447). Comme de juste, Ahmet et Amasia convoleront à Constantinople après une dernière facétie de Kéraban (p. 474-484).

Ainsi ce thème du naufrage heureux ("Der glückliche Schiffbruch", Lessing; "Il naufragio felice ", C. Questa et R. Raffaelli) ${ }^{13}$, que nos amis italiens ont suivi depuis Homère (Od. V-VI), avec Euripide (Hélène), Plaute (Rudens, mais aussi Miles Gloriosus), Mozart (Il ratto dal serraglio) et Rossini (L'Italiana in Algeri) se poursuit - avatar moins connu - avec le Kéraban-le-Têtu de Jules Verne. L'analyse d'une partie de ce roman comparée au prologue et aux deux premiers actes du Rudens a mis en évidence de très frappantes analogies, au niveau de la structure d'ensemble comme à celui des détails.

Admettra-t-on que Jules Verne s'est consciemment inspiré de Plaute? Pour ma part, j'en suis persuadé. Né en 1828 à Nantes ${ }^{14}$ dans une famille aisée de juristes et d'armateurs, le futur romancier avait fait ses humanités dès 1836 au Petit Séminaire de Saint Donatien, puis au Lycée de Nantes, et passé son baccalauréat ${ }^{15}$. Il a fort bien pu, dès sa scolarité, lire le Rudens dans le texte ou en traduction : la pièce avait tout pour le séduire. Lui-même, dès l'âge de onze ans (1839), était si épris des choses de la mer qu'il s'était embarqué sur un long-courrier en partance pour les Indes (il fut rattrapé de justesse à Paimboeuf)! Parti faire son droit à Paris (1846), il s'intéresse plus au théâtre qu'au Code Civil, se passionne pour Shakespeare, compose et fait jouer des œuvres dramatiques. C'est en 1862 seulement que Cinq semaines en ballon, publié par Hetzel avec un vif succès, orientera définitivement son inspiration vers les «Voyages extraordinaires » qui, jusqu'à sa mort en 1905 , feront sa renommée. Comment un tel homme, et sans doute dès sa prime jeunesse, n'aurait-il pas rencontré et goûté le Rudens?

\section{NOTES}

1. Collection « Le Livre de Poche Jules Verne », Hachette, 1967. Nous renverrons dans tout ce qui suit aux pages de cette édition.

2. Cf. Jules Verne, Géo hors série (2003), p. 88-99: “Mer Noire: l'Europe et l'Asie en boucle”. L'auteur de cet article, la journaliste Carole Pither, a refait en 4 x 4 le voyage de Kéraban et en a tiré aussi un livre, Sur les traces de Kéraban, éd. Stanké.

3. Qui sera une des étapes de Kéraban dans son " périple » terrestre.

4. C'est-à-dire « bohémienne » (de l'italien zingaro, -gara).

5. Sic C. QUESTA, Il ratto dal serraglio, Bologne, 1979, n. 13, p. 110.

6. Cf. pour ce destin imminent, les deux filles d'Hannon, Adelphasium et Anterastilis, dans le Poenulus.

7. Cf. F. BOLL, s.v. Fixsterne, P.W. VI 2427-2430 (l'étoile Arcturus est 盏 Boo) ; A. LE BOEUfFLE, Les noms latins d'astres et de constellations, Paris, 1977, p. 271-275. Intempéries notées par Pline, NH, II 106, 124 ; XVIII 310. 
8. On exclura le lever vespéral en février (époque du mare clausum) et le coucher matinal en juin (beau temps estival).

9. Je l'ai cherché en vain sur les trois atlas à ma disposition. Carte sommaire dans Géo (cf. n. 2), p. 97

10. Cf. E. de SAINT-DENIS, La mer dans la poésie latine, Paris-Mâcon, 1935, passim ; J. SOUBIRAN, «La météorologie à Rome : thèmes et textes ", in La météorologie dans l'Antiquité, Saint-Étienne, 2003, p. 62 sq.

11. Rd. 177-183, où Démonès apparaît sous un jour bien déplaisant, voire odieux (rudesse encore, mais moins choquante, en $R d .1202$ sq., où il maugrée devant les embrassades de la mère et de la fille réunies). Trait de mœurs (misogynie, mépris des femmes encore sensible de nos jours dans certaines régions du globe) ? Lieu commun (d'un goût douteux) de la comédie? On songe à La Fontaine (Fab. III 16) : «Je ne suis pas de ceux qui disent : "ce n'est rien : / c'est une femme qui se noie.” / Je dis que c'est beaucoup...» Jules Verne n'a eu garde d'imiter ce passage, tout au contraire : «Nous ne pouvons pas les laisser périr! » répétait Kéraban » (p. 306) « qui voulut se lancer dans ces eaux furieuses. » (p. 307).

12. Division, on le sait, non plautinienne.

13. C. QUESTA, Il ratto dal serraglio, Bologne, 1979; C. QUESTA \& R. RAFFAELLI, Maschere Prologhi Naufragi nella commedia plautina, Bari, 1984 (p. 121-144, « Il naufragio felice », par R.R.).

14. Sur les éléments biographiques qui suivent, cf. l'Appendice de l'édition citée $n$. 1 et Géo (cf. $n$. 2), p. 16 sq.

15. La culture classique de Jules Verne transparaît dans le roman. Au fil du récit, il évoque des souvenirs mythologiques (Argonautes, p. 243), historiques (retraite des Dix Mille, p. 261, et, p. 451, l'exclamation fameuse : « la mer !... la mer !...») et géographiques (notices sur l'antiquité des cités traversées, avec même des références aux auteurs grecs, Diodore de Sicile par ex.).

\section{AUTEUR}

\section{JEAN SOUBIRAN}

Université de Toulouse-Le Mirail 